its insertion by means of delicate forceps with the teeth placed at the ends of the blades. The seized portion of the iris is withdrawn from the wound and snipped off by means of thin-bladed scissors. Through the opening thus made, retained cortical lens substance is permitted to escape. The operation is termed the "modified simple" extraction. The operation was devised to avoid prolapse of the iris after simple extraction. Chandler reports 312 cases with 4 prolapses, 2 of which were the result of violence after the anterior chamber had been restored. Since the average of prolapse in simple extraction by good operators is approximately 6 per cent., the reduction by this method is very satisfactory.

\section{THE DELIVERY OF THE LENS FOR THE EX- TRACTION OF CATARACT; IRRIGATION OF THE ANTERIOR CHAMBER, AND THE FIRST TOILET OF THE WOUND.*}

\section{WEBSTER FOX, A.M., M.D.} PHILADELPHIA.

The second stage toward the successful completion in cataract operations depends, first, on the length and character of the corneal incision; second, on the laceration of the capsule or the removal of the lens within its capsule through the opening in the cornea. The importance of the amplitude of the incision is so well known to all ophthalmic surgeons that, in his symposium, Dr. Weeks has undertaken to embellish this part of our subject with the care and precision which characterize all his writings on ophthalmology. Dr. E. E. Landolt of Paris has recently written an historical review of all the work done on this subject in a monograph of 130 pages. $^{3}$ So, with Dr. Weeks on this side of the Atlantic and Dr. Landolt on the other, the ophthalmic world will be made thoroughly conversant with all that can be said or written on that delicate incision in the cornea, so important to the success of a cataract operation.

To carry out the mechanical and geometrical incision properly entails close study, for there is no operation in surgery so dependent on the cunning of the hand and its delicate training as this. Each cataract case is a law unto itself, and the operator who does not recognize immediately the necessity for a different kind of incision in patient $\mathrm{A}$ from that required in patient $\mathrm{B}$ has much to learn. No two eyes are alike; therefore, no two incisions can be mathematically the same in corner with varying geometrical areas.

With the proper incision we advance to the second stage of the operation. Shall it be with an iridectomy or without? My experience leads me to believe that if we were to operate alternately on a series of cases we would obtain far better ultimate results in at least 75 per cent. of the cases in which an iridectomy is performed, than in those in which the simple method is carried out. I have had my share of successful results by the simple method, but they have always been in selected cases.

\section{IRIDECTOMY.}

The iris may or may not present itself at the apex of the wound after the incision. It usually bulges forward, but may be replaced by gentle pressure with a Daviel

* Read In the Section on Ophtbalmology of the American Medi cal Asscclation at the Fifty-eighth Annual Session, held at At lantic City, June, 1907 .

1. La Surface de Section des Plales Faites en vue de L'Extraction de la Cataracte et de L'Iridectomie, 1905. spoon. It is then grasped with the proper iris forceps, drawn gently forward, and with one snip of the scissors is cut off close to its base. This gives the ideal keyhole iridectomy, and a space large enough for the escape of the lens without bruising the pillars of the iris, thus avoiding the complication of iritis, which is so apt to follow. In the simple operation, the hardened lens has to be forced through a space much smaller than its own diameter. This means an unusual stretching of the circular fibers of the iris; a partial paralysis is the result, which even eserin fails to hold in contraction, and is followed probably by incarceration in the lips of the wound. The currents of the fluids of the eye which flow from the wound are strongest at its apex and sweep the iris into this space like a floating flag, while the wound

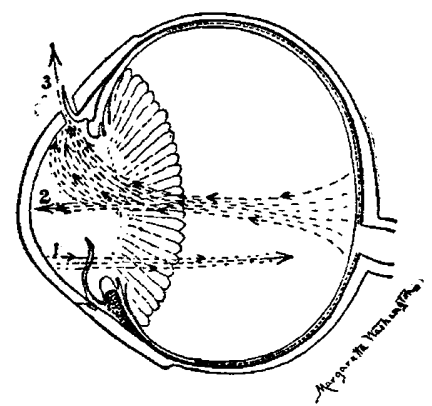

Fig. 1.-Currents of the eye, the results of pressure: 1 . Showing primal pressure toward the optic nerve. 2. Currents returning, due to counter pressure. 3. Exit through corneal incision and foating iris.

heals from its base toward the center. So long as there is the most minute opening, the currents of the eye flow rapidly or slowly according to the intraocular pressure, thus keeping the iris constantly in the gaping wound until closure takes place. In many examinations of the iris, I have found that its pupillary margin projects forward, and when this iris is bruised or stretched to the point of paralysis, it can be easily understood how the currents of the eye can sweep this floating tissue into the fissure of the incision. With this explanation it is readily understood why so few simple operations have the beautiful round pupil and the iris in its proper

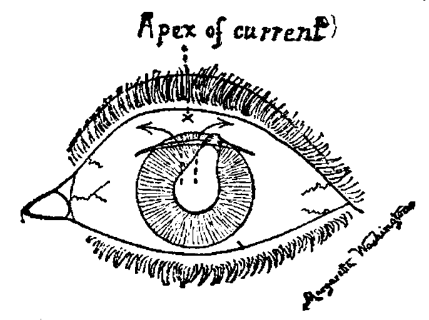

Fig. 2.-Incarceration of the iris due to paralysis of the circular fibers in simple operation.

place. The successful cases of simple cataract operations are those in which the cornea is larger and the anterior chamber is deeper than usual and the iris appears to hang perpendicularly (from its base). With all such conditions confronting one, it is not difficult to understand why, in the hands of the larger number of operators, an iridectomy is preferable.

HISTORY OF THE OPERATION BY EXTRACTION AND THE INSTRUMENTS DEVISED FOR OPENING THE CAPSULE.

To Daviel, a French surgeon, we are indebted for the operation for removing cataract by extraction as a routine practice. He performed this operation in $174 \%$, 
although it was not a new discovery, for Rliazas, in 1529 , says that "about the end of the first century Antyllus opened the cornea and drew the cataract out of the eye with a fine needle or hook," in which practice he was followed by Lathyrion. ${ }^{2}$ If history is correct, this operation was forgotten or neglected until St. Yves (170\%) performed the operation of extraction and described the operation in the following words:

When the operation of removing the cataract, which has passed into the anterior chamber, is to be performed, the patient must be seated on a chair, with the eye fairly opposed
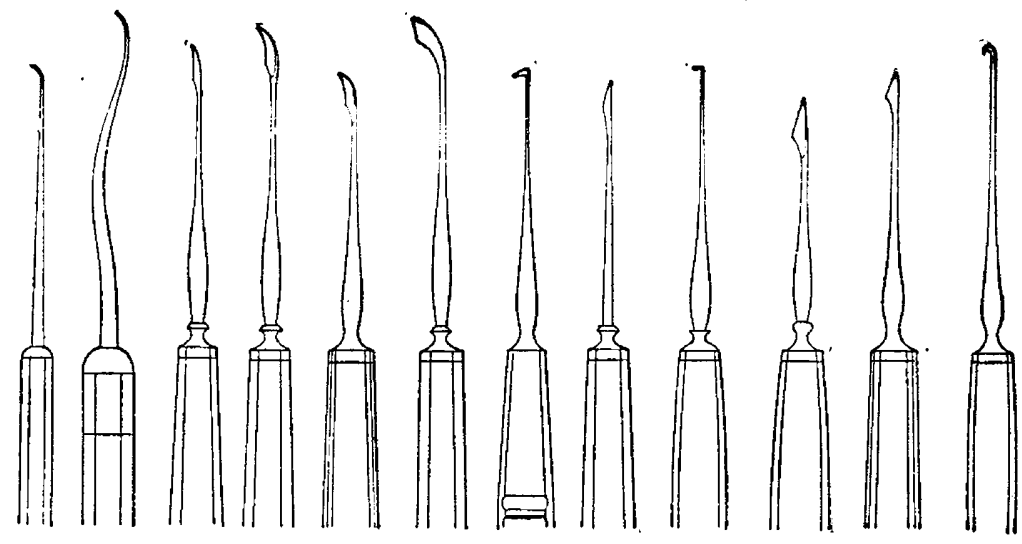

\section{2}

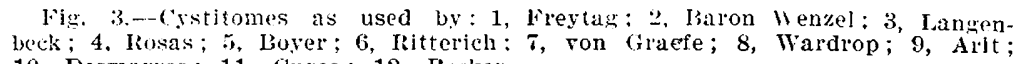
10, Desmarres; 11, Cusco; 12, Becker.

to the light; the lids are to be kept open with the thumb and forefinger of the right hand, the cornea is opened with a fine cutting lancet, commencing a little below the level of the pupil and continuing it across to the opposite edge, so as to leave only half a line on each side of the lower half of the cornea undivided. Through this opening a fine curette is to be introduced; and, by passing it behind the crystalline, its extraction is to be accomplished.

In 1708 , Petit performed an extraction by the same method. Freytag (1721) also resorted to extraction through the cornea with a "hooked needle." I'ho work of St. Yves and Petit led Daviel to follow the same method and he evidently made some improvements in the instruments of his day for the successful delivery of the cataract, for he speaks of using a small spatula (flat needle) with which he drew out from behind the pupil the different portions of the lens. Plantner (1780) speaks of extracting the lens through the corneal wound either with forceps or a little hook. From Dariel's time to the present many instruments have been devised for opening the capsule and delivering the lens, and the end is not yet. From various sources I have succeeded in gathering together almost every form of cystitome in use from Freytag, No. 1 (1721), down to the latest model of to-day. From the variety of these models, operators of 150 and more years ago were quite as ambitiops to suit their own individual methods and to be remembered by these instruments as are the surgeons of to-day.
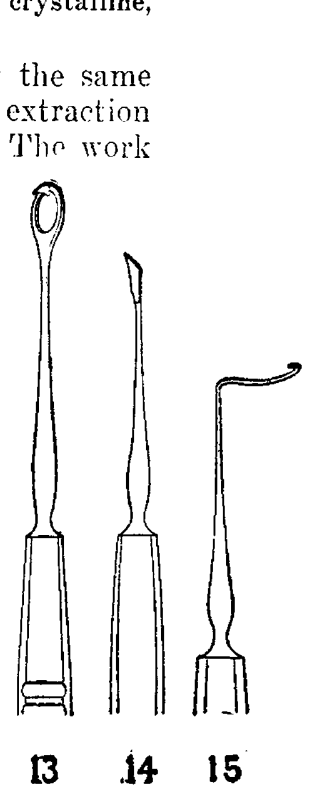
gaged in the iris, so it is the one that I have used in many cases. The instrument is passed into the anterior chamber, gently pressed against the cornea on the nasal side until it reaches the pupillary margin of the iris below, slightly turned backward and hooked into the capsule-

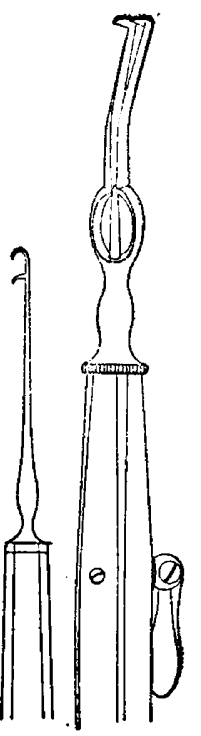
Fig. 4.-Cystitomes as used by: 13, Rettremieux; 14, Knapp; 15, Jaeger not too deeply-then drawn upward toward the top, lacerating the capsule in its upward movement. It is again passed downward through this same opening, passing across horizontally until the iris boundary is again reached, a second vertical tear is made to the top across to the first incision. In this way a rectangular opening of a large size is made in the anterior 
capsule. Meyer attempted this same operation with the double cystitome invented by himself (Fig. 4, 17). This instrument is introduced in the ordinary way into the anterior chamber; on arriving at the inferior margin of the pupil, by pressing a lever, the cystitome becomes double; and, on drawing it back toward the corneal margin, a large central opening is made in the capsule. Before withdrawing it from the wound it is closed and this brings out the capsular flap. This instrument never became very popular, and now with many others is only an ophthalmic curiosity. While in Heidelberg I saw Becker use a cystitome (Fig. 3, 12) which bears his name and he used it most successfully. Weber (Fig. 4, 16) improved this instrument by devising a double hook, the fine teeth of which are placed one beneath the other. "He moves it in the capsule from one side of the pupil to the other and from the two angles of the capsular wound toward the incision in the cornea. If the capsular flap remains attached to the hook it is snipped off with the scissors." Geyet proposed to open the capsule near its superior borders by slight pressure. He tilts the margin of the lens into the corneal wound and then opens it with a small linear knife. Trousseau and some other dexterous surgeons pass the point of the knife through the capsule in making the corneal incision. This is not so difficult as would appear at first sight. It is done in the following way: Point the knife toward the tip of the nose, and, as soon as the instrument has entered the anterior chamber, pass the iris, dip the point of the blade into the capsule, then throw the handle backward and downward; this raises the point of the knife, lacerating the capsule and making the counter puncture in the cornea. Knapp (Fig. 4, 14) also adopted the peripheral incision with a cutting cystitome, and by his teaching both his instrument and method have been extensively followed. This cystitome is very similar to that used by Boyer (Fig. 3, 5), who lived during the eighteenth century. Landolt (Fig. 4, 18) has devised a right-and left-hand cystitome adapted for each cye, but at convenient angles to be inserted in the anterior chamber. Ziegler (Fig. 4, 19) has devised a lance-shaped cystitome which I have found extremely useful in making a large cut in a thickened capsule. The flexible cystitome of to-day (Fig. 3, 7) with its small-cutting lance-shaped blade was used by each and every surgeon at Moorfields in Iondon during my term of service there, and it is probably used by more ophthalmic surgeons than is any other form. De Wecker (Fig. 5, 1). Foster (Fig. 5, 2), Collins (Fig. 5, 3), Licbreich (Fjg. 5, 4) have substituted capsular forceps specially devised for grasping and extracting as much of the anterior capsule as possible. This method of opening the capsule requires great delicacy of touch and immobility of the eye. It is not my province nor wish to speak about the merits and demerits of each and every one of the many crstitomes made; I have only mentioned a few which I have found the most mechanically perfect and best adapted to lacerating the capsule.

After the capsule has been opened by any one of the instruments described, cystitomes or forceps, the next stage is the delivery of the lens. Pressure on the lower half of the cornea and gentle pressure against the upper lip of the incision causes the eye currents to move backward in the lower half of the eye and forward in the upper half of the globe in direct ratio to this pressure and counter-pressure. The lens (Fig. 6) is thus made to tilt forward on its equatorial axis and by continued gentle pressure below it is forced forward and upward with the currents finding their way through the incision, and thus the cataract is gently expressed. If the pressure is too great, caused by the heavy hand of the operator, or a spasm of the muscles of the globe or eyelids, the lens is forcibly expelled and a gush of vitreous is sure to follow. The latter result was experienced during an operation for extraction, performed before the senior class in the amphitheater of the Medico-Chirurgical College. The eye had been thoroughly anesthetized and the patient was passive, yet, when the incision was made, an involuntary spasm of the orbicularis muscle tonk place forceful enough to close a Clark stop speculum. The force exerted was so great that the lens was thrown above my head and fell on the floor some distance from the patient. In another case the force exertcd was not so great and the lens did not fly more than six inches from the eyeball. Some operators use the fixation forceps as a means of exerting counter-pressure, but any one thinking for a moment of the effect and counter-effect of this force on the fiuids of the eye must at once realize the cause of many failures in expelling the lens. This is explained (Figs. 1 and 2), first, as to the effect of pressure on the lower third of the eye; second, the cause of a one-sided prolapse of the iris when the pressure is not

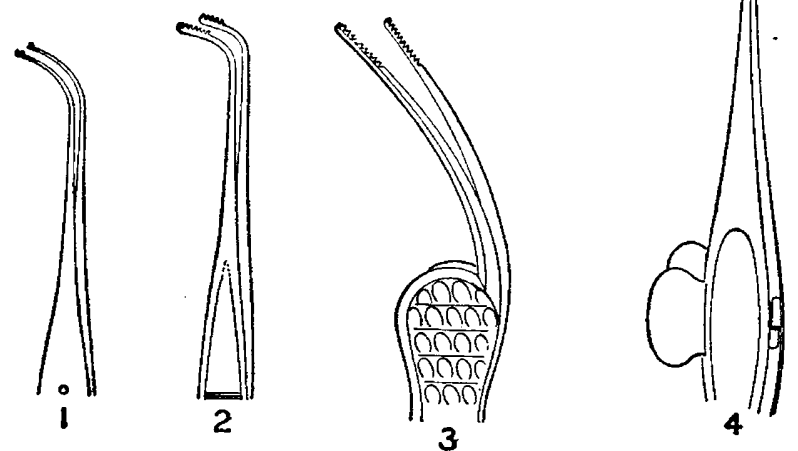

Fig. 5.-Capsular forceps as used by : 1, De Wecker, 2, Foster ; 3 , Collins; 4, Liebreich.

properly exerted on the eyeball directly in the median line.

THE REMOVAL OF CORTICAI, SUBSTANCE.

Is it deleterious to allow cortical substance to remain? I think it is to a certain extent. My observation has been that the posterior capsule always grows denser, and, in consequence, is more obstructive to vision when the tenacious lenticular masses are allowed to remain in the anterior chamber. I use massage first, and the Daviel curette second, then follow this by irrigation, if I have not cleared the anterior chamber sufficiently by the two methods mentioned. As soon as the cataract has been expressed the speculum is removed. Pressure is then brought to bear against the upper eyelid with the thumb of the left hand as a fixed point, and with the tip of the little finger of the right hand, the lower lid is gently pressed against the lower third of the cornea. Then, with an upward movement through the medium of the lids, all extraneous matter possible is forced out through the lips of the wound. This action usually cleanses the anterior chamber, leaving the pupil a velvet black and the iris in its proper position. Sometimes 
with this massage it is not always possible to clear the pupillary space nor the anterior chamber from all cortical substance; the curette is then called into use.

The curette has a limited field and removes only such particles that lie in the pupillary area, it being unsafe to pass the curette behind the iris and into the folds of the posterior capsule. Massage and the curette are only safe in the combined operation; the latter is not applicable in the simple operation. If I find that the pupillary space is black and the pillars of the iris stand out clear I let well enough alone and close the eye; but, if the anterior chamber presents a hazy appearance, I resort to irrigation with a physiologic salt solutionthe mercuric salts or distilled water should not be used, as both have a deleterious effect on the endothelium of the anterior chamber. I have in my possession various irrigators-McKeown's, Lippincott's, Elliott's and the simple pipette of Terson. The most practical and safest is McKeown's, for with this instrument the fluid reaches every part of the interior of the capsule and forces out with the current all extraneous material and the cornea assumes its normal convexity.

I am firmly convinced, however, that the fewer instruments placed in the anterior chamber the better, but when necessity compels, the methods I have suggested are, I believe, the safest and best.

\section{EXTRACTION OF THE CATARACT WITHIN THE CAPSULE.}

From incomplete knowledge of the earliest operations, and from the models of instruments that have come down to us through the centuries, it is safe to say that whenever the cataract was displaced, first, by depression, and second, through the cornea, it was taken out of the line of vision within its capsule. I shall not deal with the couching method, but simply mention the extraction of the cataract through the cornea within its capsule. The first surgeons who succeeded by this method were Freytag (1721), Janin, Heckel and Richter, about the year 1773. A little later Mohrenheim, and especially Beer (1799), advised this method. It was again revised by Christiaen (1845), who strongly recommended it. Then followed Sperino, Gioppi, Feneglio, Gradenigo, Rosmini and Alexander Pagenstecher. The latter, from 1865 to 1875 , performed 353 operations, and his work was given to the world in an article published by $\mathrm{D} r$. Hermann Pagenstecher, his brother, in $18 \% \%$.

Delgado of Madrid attempted to extract the cataract in its capsule in the following manner:

He begins by introducing into the anterior chamber, as in discission per corneam, an instrument combining the needle with a spatula; with this he detaches the lens and makes it mobile by gentle pressure on the periphery of the cataract. Having done so, he withdraws the instrument, waits until the aqueous has again filled the anterior chamber, and then extracts the lens in its capsule, using von Graefe's peripheral incision and the superior iridectomy.

From the days of Pagenstecher and Delgado until the present day many operators have had cases in which, owing to a thickened capsule and atrophy of the suspensory ligament (zonule of Zinn), they have delivered the cataract within its capsule without difficulty. Encouraged by this, they have operated on patients in whom these conditions did not exist and have not met with the same brilliant results.

For several years past, whenever I have found a hardened cataract with a thickened capsule and atrophied suspensory ligament, I have dislocated the lens with a Jaeger's cystitome (Fig. 4, 15) and then extracted the lens within its capsule. My method is as follows: Pass- ing the instrument into the anterior chamber and hooking the lens at its inferior margin I make a backward pass, at the same time pressing the upper lip of the incision very gently downward and backward; this tilts the upper part of the lens forward; the lens is then brought back into its normal position, and this is followed by rotation on its axis, done by hooking the cystitome in the capsule. Then, removing the cystitome and by counter pressure on the lower third of the cornea with a tortoise shell spatula, I express the cataract through the incision without loss of vitreous. During the last five years I have operated on at least fifty patients by this method, and I find it preferable to the method advised by those operators who express the lens within its capsule by pressure. In the last ten cases, in which I followed the method suggested by Pagenstecher, Smith, etc., I have found considerable excitation of the iris, more or less pronounced irritability in healing, the eyeball remaining red, and there is also much distortion of the iris. Among the Anglo-Saxon race I am certainly not in favor of the operation of extracting the lens within its capsule as a routine method. Even in those cases in which I have delivered the lens within its capsule and in which the suspensory ligament was atrophied and no secondary inflammation followed, the average visual acuity obtained was no better than in those cases in which the ordinary operation was performed.

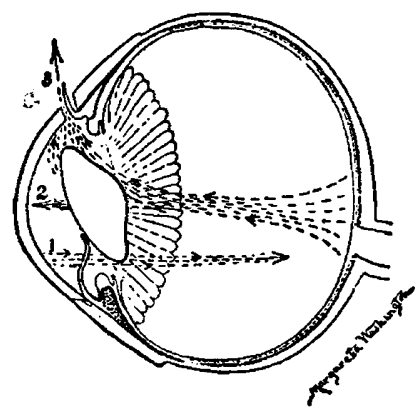

Fig. 6.-Demonstrating the forces which expel the crystalline lens: 1. Primal pressure with spatula on cornea. 2. Currents returning, tllting lens. 3. Exit of crystalline lens through corneal incision.

I have stated that the openings of the capsule are many and varied, and so are the instruments to make these openings. Each cataract is surrounded by a pathologic capsule. This change may be invisible to the naked eye, or very much thickened and dense and easily detected by oblique illumination, and yet it is very difficult to recognize the different types. Diaphanoscopy does not help us, oblique illumination may in some cases, but it is not to be relied on. I have found that the age of the cataract and the senility of the individual are the best guides. A cataract of one year's growth has a capsule with a bluish cast; a cataract of two years' growth has a capsule with a pale color, moderately dense in appearance; a cataract of three years' growth and over, has a capsule of yellow cast, and is dense and inelastic. As the cataract matures the capsule thickens, while the zonule of Zinn, or suspensory ligament, grows thinner or degenerates. I have found this classification of cataracts on the whole fairly accurate.

\section{DISLOCATION AND MALPOSITION OF THE LENS.}

Occasionally a dislocation of the lens occurs when the corneal incision is made or when the incision is not long enough and too much pressure is exerted in the center instead of in the lower third of the cormea, forcing the 
lens backward instead of tilting it forward. Sometimes a prolapse of vitreous takes place after the incision of the iris or after the laceration of the capsule. This is an evidence of a rupture of the zonule of Zinn and a partial dislocation of the lens. The speculum should be removed at once, the patient allowed to rest quietly for a few minutes, the eyelids then separated by a trustworthy assistant and the lens immediately extracted by means of a scoop (Waldau "Schuft," Critchett's, Bowman's or Irevis's). If there is much loss of vitreous and the eyeball becomes flaccid, the eyelids are separated and the eveball, through the anterior chamber, is filled with a tepid physiologic salt solution.

\section{ECTOPIA LENTIS.}

In rare instances we meet with a congenital displacement of the crystalline lens upward or downward to the nasal or temporal side. When we meet with cataracts of this character it would be unwise or dangerous to make a corneal incision and attempt to extract the lens through the opening with a scoop; the vitreous is fluid and would certainly escape in large quantities, causing a total collapse of the eyeball and consequent loss of vision. In such cases it is safer to follow the couching method, for it is almost impossible to extract the cataract by the ordinary method and at the same time preserve the integrity of the eyeball.

\section{LENS IN ANTERIOR CHAMBER.}

The crystalline lens may find lodgement in the anterior chamber and cause an irritation to the iris and ciliary body. I have found that when the dislocated lens remains in the anterior chamber for more than a year it solidifies and becomes a calcarcous mass without an increase or loss in its size. It then becomes a serious menace to the eye by causing iritis or iridocyclitis, but the greater danger lies in its provoking sympathetic ophthalmia. To remove this foreign body I make an incision through the horizontal plane of the cornea. The puncture is made near the outer side of the cornea directly into the anterior chamber, the point of the knife is passed along until the foreign body is approached, then the point of the blade is passed over it and beyond to the point of counter puncture, thus making a long incision through the center of the cornea. The speculum is then removed and the anterior chamber opened by pressing the partially closed eyelids with the thumbs of the right and left hand. If the calcified lens can not be forced out in this manner a small hook may be passed through the incision back of the lens while the pressure is maintained with the thumb of the right hand above and the lens then drawn out. By this method I have successfully removed the lens in five cases.

\section{LOSS OF VITREOUS.}

Is the loss of vitreous, in large and small quantities, detrimental to the success of the operation? The surgeon of to-day looks on the loss of vitreous with great seriousness and aims to prevent it at all hazards. Some of the older authorities did not take the loss of vitreous with much distrust, for Lawrence says:

The escape of vitreous humor is not to be regarded in itself as a very serious circumstance. The space which it before occupied is filled by aqueous secretion. There is no doubt that one-fourth or one-third, and perhaps even one-half, of the vitreous humor may be lost in this way without interfering with the result of the operation. In many instances it seems rather to contribute to success; it lessens the bulk of the globe, and thus prevents the tension which occasionally succeeds the operation. Sometimes there is a spasmodic action of the muscles, propelling the vitreous humor against the cornea and preventing the apposition of the flap. I have in such cases introduced the curette through the pupil and let out some of the vitreous humor purposely.

In my opinion it should be our aim to deliver the lens without loss of vitreous. I have had a few cases in which at least one-fourth of the vitreous was lost, but instead of allowing the globe to remain in the flaccid condition, which this loss of vitreous would entail, I have immediately filled the eyeball with a tepid saline solution (physiologic salt solution) and have had good recovery ensue. When this has not been done and the condition has been left to Nature, subacute glaucoma has almost invariably followed. It is only fair to state, however, that in these cases the tension was slightly above normal before operating and that glaucoma, in an insidious form, may have already existed. For a trifling loss of vitreous no heed need be paid other than a careful snipping of this bead from the lips of the wound and a gentle backward stroking of the edges of the incision. If at the end of twenty-four hours there be again a protrusion of vitreous it should be cut off and the lips of the wound touched with a 1 per cent. solution ( 5 grains to the ounce) of silver nitrate. When the vitreous humor has its normal consistency prolapsus rarely follows the first cleansing of the wound immediately after the operation. It is impossible to prevent prolapsus in those cases in which the vitreous has degenerated and become fluid. ITere it may be necessary even to go so far as to stitch the opening with two stitches of silk thread to prevent leakage, as I have done on several occasions. If we could foresee this condition-but I know of no physical sign by which we can discover the fluid vitreous-no surgeon would follow the ordinary method of operating in such cases. If we could be sure of our premises the only justifiable operation here would be the couching method.

To-day the ophthalmic world is slowly turning toward the Orient and watching with interest the work done by Surgeon Major Henry Smith, I. M. S., in delivering the lens within the capsule. In such a prolific field as India, with its three hundred millions of inhabjtants, an ophthalmic surgeon has unlimited opportunities to put into practice any method to which he aspires. If one surgeon can perform 2,616 extractions in one year, with the following results, iritis 0.3 per cent., escape of vitreous 6.8 per cent., capsule bursting 8 per cent., capsule left behind 4.28 per cent, first class results 99.28 per cent., second class results 0.3 per cent, failures 0.34 . per cent., it must make the ophthalmic surgeons of Europe and America hesitate to give their results drawn from the meager field around them. My experience leads me to agree with Surgeon Major H. Herbert, I. M. S., of Bombay, who says: "Extraction within the capsule is, with us, commonly reserved for over-ripe cataracts in which the capsule is not only opaque, but also too tough to be torn by the cystitome." He further says : "I have been surprised and disappointed to find the average visual acuteness obtained by this operation, tested by spherical lenses on discharge from the hospital about a fortnight after the operation, to be certainly no better, and, perhaps, rather inferior to that obtained by the ordinary operation." In commenting on the work done by these two operators of equal ability in the same field, with the same race, we have diametrically opposite opinions as to the value of the operation of removing the lens within its capsule.

Neyer of Paris, one of the most slillful ophthalmic 
surgeons of his day, in speaking of this operation twentyfive years ago, said:

Extraction of the lens in its capsule does not appear to be admissiule as a general method applicable to all cases, but should be restricted to those in which it seems probable that the strength of the suspensory ligament is defeative-for example, when the cataract is over-ripe, when the iris is trem. ulous, and in strongly myopic eyes in which a general dis. tension of the globe exists.

Macnamara says:

This is by no means a new method of extraction of the lens, having been practiced with varying success since $17 \% 5$ and strongly advocated by Pagenstecher and M. Sperino. Having performed this operation extensively since 1864, I am convinced that if it were possible in every case in which we operate to remove the lens in the capsule without damag. ing the other structures of the eye, we should have reached perfection in the extraction of the cataract.

Dr. D. W. Green, ${ }^{3}$ Dayton, Ohio, in a recent article, his given his experience in operating in twenty-four cases by the so-called "Indian method." He says:

Is it an operation that ought generally to be adopted, as Smith contends it should be, by the average operator and in the average class of cases? I thirk not, for the following reasons: It is more difficult to deliver the lens in this way than with the capsulotomy, even if one be experienced with the method. The operator who only does ten or twclve operations a year or fewer can not from this small number acquire enough skill to do justice to the method himself.

In referring to Dr. Green's statistics his results were not encouraging.

The lens was delivered entire in 55 per cent.; vitreous was lost in 35 per cent.; glaucoma followed in 15 per cent.; iritis in 20 per cent.; and an average of $20 / 70$ vision was secured with correcting glasses.

\section{WIIAT METHOD SHOULD WE ADOPT?}

From the recorded experiences of many ophthalmic surgeons no doubt the conscrvative instructors in ophthalmology would select the combined operation as the method to be taught in our schools of ophthalmology for the younger ophthalmologists to follow. The combined operation, in the hands of the majority of operators, good, bad and indifferent, the world over, gives a larger number of perfectly successful results, and cases of complete failures are less frequent than by any other.

\section{PREPARATION OF INSTRUMENTS.}

Formerly, I took all the precautions necessary for thorough sterilization by boiling in water and by dry heat in a sterilizer; these extra precautions I found resulted in destroying instruments and did not improve my statistics. I long ago (ten years) returned to cleansing my instruments in diluted alcohol (alcohol $3 \mathrm{i}$, aquæ destillata 5 iii); after immersing them in this solution for five minutes they are then carefully dried with sterilized gauze.

Here $I$ wish to enter a protest against the introduction of metallic handles for knives and cystitomes as a substitute for ivory. An instrument having a metallic handle can not be held by fingers and thumb with the same lightness and delicacy of touch as one having an ivory handle. In an operation so important as the removal of cataract, what conscientious surgeon hesitates to obtain the best instruments of the choicest mechanical finish to aid him in making such operations a surgical success, especially since we have the means of making these refined instruments clean, preserving their cutting edge,

3. Ophthalmology, January, 1907. and not destroying them by heat and chemicals before their work is accomplished?

Drs. Weeks and Landolt have called attention to the incision for the easy delivery of the cataract. I have described the accuracy of manipulation of the iris in securing the ideal keyhole iridectomy; the opening of the capsule, by many instruments devised for the purpose, vertical and horizontal crosses, peripheral incisions, and extracting the anterior leaf with capsular forceps; the delivery of the lens within its capsule and without, not even forgetting the flushing of the anterior chamber to clear this space of all cortical matter or filling the globe with a saline solution where much vitreous has been lost. All this should be done with a gentleness of touch and with delicate instruments "so that nothing be cffected by force which can be accomplished by art."

[The other papers in tilis Sympositm on Cataract will BE PLDLISIIED NEXT WEEI, AND TIIE DISCUSSION WILL FOLLOW.]

\section{SUBSEQUENT HISTORIES OF ONE HUNDRED AND SIXTY “ARRESTED CASES" OF TU- BERCULOSIS TREATED AT THE SHAR- ON SANATORIUAI, 1891-1906.* \\ VINCENT Y. BOWDITCH, M.D. BOSTON.}

With the Coliaboration of Walter A. Gimfix, M.D. SHARON, MASS.

In offering you the results of work of over fifteen years at the Sharon Sanatorium, this time with the invaluable assistance of Dr. Griffin, neither he nor I offer any apology for what may seem an unnecessary harping on a subject which has become familiar in the past few years since the crusade against tuberculosis has assumed such great proportions.

It must be remembered that in certain respects, so far as climate, altitude and proximity to the sca and a large city are concerned, the Sharon Sanatorium was for several years unique. The results of treatment, therefore, should be kept before you that you may judge whether that which was begun sixteen years ago as an experiment has proved a success or otherwise.

As shown by the title of the paper, I still adhere to the term "arrested" in speaking of cases in which the most important symptoms (chiefly cough, expectoration and fever) have disappeared during a long or short stay at the sanatorium, the patient's general appearance being one of good health. This is chiefly done for the sake of uniformity with previous records given to this society, ${ }^{1}$ although in the annual reports of the institution the nomenclature recommended by the National Association for the Study and Prevention of Tuberculosis has been added to the older method. A feeling of conservatism, moreover, and a desire to counteract the harmful effect of claiming too much at first in the treatment of a disease so uncertain and treacherous as tuberculosis, are additional reasons, I confess, for not having made use even of the term "apparently cured" when the patients leave the sanatorium, in spite of the fact that

- Read at a meeting of the American Climatological Association, at Washington, D. C., May, 1907.

1. Subsequent Histories of Arrested Cases of Phthisis treated at the Sharon Sanatorium. Boston Med. and Surg. Jour., June 22, 1899 ; Trans. Amer. Climatologleal Assn., 1899. Subsequent Histories of Seventy-nine Arrested Cases of Phthisis treated at the Sharon Sanatorium from 1891 to 1902 . THE Jocrnal A. M. A., Nov. 14, 1903; Trans. Amer. Cllmatological Assn., 1903. 